\title{
COHOMOLOGY OF THE COMPLEMENT TO AN ELLIPTIC ARRANGEMENT
}

\author{
A. LEVIN $^{\star}$, A. VARCHENKO $\diamond$ \\ * State University - Higher School of Economics, Department of Mathematics, \\ 20 Myasnitskaya Street, Moscow, 101000, Russia \\ * Laboratory of Algebraic Geometry, GU-HSE, 7 Vavilova Street, Moscow, 117312, Russia \\ $\diamond$ Department of Mathematics, University of North Carolina at Chapel Hill \\ Chapel Hill, NC 27599-3250, USA
}

\begin{abstract}
We consider the complement to an arrangement of hyperplanes in a cartesian power of an elliptic curve and describe its cohomology with coefficients in a nontrivial rank one local system.
\end{abstract}

\section{INTRODUCTION}

We start with a cartesian power $E^{k}$ of an elliptic curve $E$ and a nontrivial rank one local system on $E^{k}$. We consider an arrangement of elliptic hyperplanes in $E^{k}$ and describe the cohomology of its complement with coefficients in the local system. We show that the cohomology is nontrivial only in degree $k$. We present each cohomology class by a unique closed holomorphic differential form. Our forms are elliptic analogs of the Arnold-Brieskorn-OrlikSolomon logarithmic differential forms representing cohomology classes of the complement to an arrangement of hyperplanes in an affine space. For the elliptic discriminantal arrangement our forms are the forms considered in [FV1, FV2, FRV] to solve the KZB equations in hypergeometric integrals and to construct Bethe eigenfunctions to the elliptic Calogero-Moser operators.

To simplify the exposition, we first consider in Sections 2 and 3 the case of an elliptic discriminantal arrangement, then in Sections 4 and 5 we consider arbitrary elliptic arrangements.

The authors thank the Max Planck Institute for Mathematics in Bonn for hospitality.

\section{Cohomology of An Elliptic Discriminantal ARRANGEMENT}

Fix a natural number $k$ and $\tau \in \mathbb{C}, \operatorname{Im} \tau>0$. Denote $\Lambda=\tau \mathbb{Z}+\mathbb{Z} \subset \mathbb{C}$. The group $\Gamma=\mathbb{Z} \oplus \mathbb{Z}$ acts on $\mathbb{C}$ by transformations $(l, m): t \mapsto t+l \tau+m$. The action on each factor gives an action of $\Gamma^{k}$ on $\mathbb{C}^{k}$. Denote by $p: \mathbb{C}^{k} \rightarrow \mathbb{C}^{k} / \Gamma^{k}$ the canonical projection onto the space of orbits. We have $\mathbb{C}^{k} / \Gamma^{k}=E^{k}$, where $E$ is the elliptic curve $\mathbb{C} / \Gamma$.

For each representation $\rho$ of $\Gamma^{k}$ on a vector space $W$ we get a vector bundle over $E^{k}$ with a flat connection, which is $\left(\mathbb{C}^{k} \times W\right) / \Gamma^{k} \rightarrow \mathbb{C}^{k} / \Gamma^{k}$. In particular, we may fix complex numbers

\footnotetext{
^ Supported in part by AG Laboratory GU-HSE, RF government grant, ag. 11 11.G34.31.0023

${ }^{\star}$ Supported in part by NSF grant DMS-1101508
} 
$\boldsymbol{w}=\left(w_{1}, \ldots, w_{k}\right)$, take $W=\mathbb{C}$, and $\rho_{\boldsymbol{w}}(\gamma)=e^{2 \pi \sqrt{-1}\left(w_{1} l_{1}+\cdots+w_{k} l_{k}\right)}$ for $\gamma=\left(l_{1}, m_{1}\right) \times \cdots \times$ $\left(l_{k}, m_{k}\right)$. This line bundle over $E^{k}$ with the flat connection will be denoted by $\mathcal{L}_{\boldsymbol{w}}$.

We say that the numbers $\boldsymbol{w}=\left(w_{1}, \ldots, w_{k}\right)$ are discriminantal convenient if for any subset $I \subset\{1, \ldots, k\}$ the sum $\sum_{i \in I} w_{i}$ is not in $\Lambda$.

Fix distinct complex numbers $\boldsymbol{z}=\left(z_{1}, \ldots, z_{n}\right)$. The discriminantal arrangement $\mathcal{C}_{\boldsymbol{z}}$ in $\mathbb{C}^{k}$ with parameters $\boldsymbol{z}$ is the arrangement of hyperplanes:

$$
\begin{array}{ll}
H_{i}^{a}: t_{i}-z_{a}=0, & i=1, \ldots, k, a=1, \ldots, n ; \\
H_{i j}: t_{i}-t_{j}=0, & 1 \leqslant i<j \leqslant k .
\end{array}
$$

Let $M_{\boldsymbol{z}}$ denote its complement $\mathbb{C}^{k}-\cup_{H \in \mathcal{C}_{\boldsymbol{z}}} H$.

The $\Gamma^{k}$-orbit of $\mathcal{C}_{\boldsymbol{z}}$ is the infinite arrangement $\mathcal{C}_{\boldsymbol{z}, \Gamma^{k}}=\left\{\gamma(H) \mid \gamma \in \Gamma^{k}, H \in \mathcal{C}\right\}$. Denote by $M_{\boldsymbol{z}, \Gamma^{k}}$ its complement $\mathbb{C}^{k}-\cup_{\gamma \in \Gamma^{k}, H \in \mathcal{C}_{\boldsymbol{z}}} \gamma(H)$. Denote by $\tilde{M}_{\boldsymbol{z}, \tau} \subset E^{k}$ the image of $M_{\boldsymbol{z}, \Gamma^{k}}$ under the projection $p$.

Theorem 2.1. Assume that the numbers $\boldsymbol{w}$ are discriminantal convenient and $z_{1}, \ldots, z_{n}$ project to distinct points of $E$. Then $H^{\ell}\left(\tilde{M}_{\boldsymbol{z}, \tau} ; \mathcal{L}_{\boldsymbol{w}}\right)=0$ for $\ell \neq k$ and $H^{k}\left(\tilde{M}_{\boldsymbol{z}, \tau} ; \mathcal{L}_{\boldsymbol{w}}\right)$ is canonically isomorphic to $H^{k}\left(M_{\boldsymbol{z}} ; \mathbb{C}\right)$, the $k$-th cohomology group with trivial coefficients of the complement in $\mathbb{C}^{k}$ to the discriminantal arrangement.

Here $H^{*}\left(\tilde{M}_{\boldsymbol{z}, \tau} ; \mathcal{L}_{\boldsymbol{w}}\right)$ denotes the cohomology of $\tilde{M}_{\boldsymbol{z}, \tau}$ with coefficients in the local system of horizontal sections of $\mathcal{L}_{\boldsymbol{w}}$. Theorem 2.1] is proved in Section 5 ,

The space $\tilde{M}_{\boldsymbol{z}, \tau}$ is a $K(\pi, 1)$-space and our theorem describes the cohomology of the fundamental group of $\tilde{M}_{\boldsymbol{z}, \tau}$ with coefficients in $\mathcal{L}_{\boldsymbol{w}}$. Notice that the fundamental group of $\tilde{M}_{\boldsymbol{z}, \tau}$ is a subgroup of the pure elliptic braid group.

The cohomology $H^{k}\left(M_{\boldsymbol{z}} ; \mathbb{C}\right)$ of the complement to the discriminantas arrangement in $\mathbb{C}^{k}$ are presented be explicit logarithmic forms by the Arnold-Brieskorn-Orlik-Solomon theory. Below we describe logarithmic differential forms representing elements of $H^{k}\left(\tilde{M}_{\boldsymbol{z}, \tau} ; \mathcal{L}_{\boldsymbol{w}}\right)$. Those forms were used in [FV1, FV2, FRV] to give integral hypergeometric representations for solutions of the KZB equations with values in a tensor product of highest weight representations of a simple Lie algebra and to construct Bethe eigenfunctions of elliptic Calogero-Moser operators.

\section{DifFEREntial FORMS OF A Discriminantal ARRANGEMENT}

In this section we follow [FRV]. Theorem 3.3 is new.

3.1. Combinatorial space. An ordered $k$-forest is a graph with no cycles, with $k$ edges, and a numbering of its edges by the numbers $1,2, \ldots, k$. We consider the ordered $k$-forests on the vertex set of symbols $\left\{z_{1}, \ldots, z_{n}, t_{1}, \ldots, t_{k}\right\}$. An ordered $k$-forest $T$ is admissible if all $t_{1}, \ldots, t_{k}$ are among the vertices of $T$ and each connected component of $T$ has exactly one vertex from the subset $\left\{z_{1}, \ldots, z_{n}\right\}$.

Let $\mathcal{A}_{n}^{k}$ be the complex vector space generated by the admissible ordered $k$-forests, modulo the following relations:

R1: $T_{1}=-T_{2}$ if $T_{1}$ and $T_{2}$ have the same underlying graph, and the order of their edges differ by a transposition; 
R2:

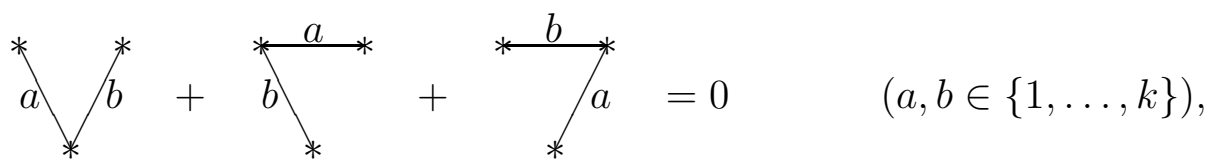

that is, the sum of three $k$-forests that locally (i.e. their subgraphs spanned by 3 vertices) differ as above, but are otherwise identical, is 0 .

A linear map $\phi$ of $\mathcal{A}_{n}^{k}$ to a vector space $W$ is called a representation of $\mathcal{A}_{n}^{k}$. Suppose we are given a vector space $W$ and a vector $\phi(T) \in W$ for every admissible $k$-forest $T$. This data induces a representation if the assignment $T \mapsto \phi(T)$ respects relations $\mathrm{R} 1$ and $\mathrm{R} 2$.

3.2. Rational representation. Let $e$ be an edge of an admissible forest $T$. The connected component of $T$, containing $e$, has exactly one vertex, say $z_{a}$, from the set $\left\{z_{1}, \ldots, z_{n}\right\}$. Denote by $h(e)$ and $t(e)$ the head and tail of the edge $e$, i.e. the vertices adjacent to $e$, farther resp. closer to the vertex $z_{a}$.

Fix distinct complex numbers $\boldsymbol{z}=\left(z_{1}, \ldots, z_{n}\right)$. To an admissible forest $T$ with ordered edges $e_{1}, \ldots, e_{k}$, we assign a closed holomorphic differential $k$-form $\phi_{\text {rat }}(T)$ on $M_{\boldsymbol{z}}$ by the formula

$$
\phi_{\text {rat }}(T)=\wedge_{i=1}^{k} d \log \left(h\left(e_{i}\right)-t\left(e_{i}\right)\right) .
$$

This assignment defines a representation of $\mathcal{A}_{n}^{k}$ on the space of $k$-forms on $M_{\boldsymbol{z}}$, see [A, OS]. By [A, OS], the representation is an isomorphism onto its image, see Proposition 2.1 in [FRV]. We denoted the image by $\mathcal{A}_{\boldsymbol{z}}^{k}$. The assignment to a form its cohomology class gives a linear map $\mathcal{A}_{n}^{k} \rightarrow H^{k}\left(M_{\boldsymbol{z}} ; \mathbb{C}\right)$.

Theorem $3.1([\mathrm{~A}, \mathrm{OS}])$. The map $\mathcal{A}_{n}^{k} \rightarrow H^{k}\left(M_{\boldsymbol{z}} ; \mathbb{C}\right)$ is an isomorphism.

3.3. Theta representation. For $z, \tau \in \mathbb{C}, \operatorname{Im} \tau>0$, the first Jacobi theta function is defined by the infinite product

$$
\begin{gathered}
\theta(z)=\theta(z, \tau)=\sqrt{-1} e^{\pi \sqrt{-1}(\tau / 4-z)}(x ; q)\left(\frac{q}{x} ; q\right)(q ; q), \\
q=e^{2 \pi \sqrt{-1} \tau}, x=e^{2 \pi \sqrt{-1} z}, \quad(y ; q)=\prod_{j=0}^{\infty}\left(1-y q^{j}\right),
\end{gathered}
$$

[WW]. It is an entire holomorphic function of $z$ satisfying

$$
\theta(z+1, \tau)=-\theta(z, \tau), \quad \theta(z+\tau, \tau)=-e^{-\pi \sqrt{-1} \tau-2 \pi \sqrt{-1} z} \theta(z, \tau), \quad \theta(-z, \tau)=-\theta(z, \tau) .
$$

By $\theta^{\prime}(z, \tau)$ we will mean the derivative in the $z$ variable. Define

$$
\sigma_{w}(t)=\sigma_{w}(t, \tau)=\frac{\theta(w-t, \tau)}{\theta(w, \tau) \theta(t, \tau)} \cdot \theta^{\prime}(0, \tau) .
$$

The listed properties of the theta function yield that the function $\sigma$-viewed as a function of $t$ - has simple poles at the points of $\Lambda \subset \mathbb{C}$, as well as the properties

$$
\sigma_{w}(t+1, \tau)=\sigma_{w}(t, \tau), \quad \sigma_{w}(t+\tau, \tau)=e^{2 \pi \sqrt{-1} w} \sigma(t, \tau), \quad \operatorname{Res}_{t=0} \sigma_{w}(t, \tau)=1 .
$$

We also have

$$
\sigma_{w_{1}+w_{2}}(t-u) \sigma_{w_{2}}(s-t)-\sigma_{w_{2}}(s-u) \sigma_{w_{1}}(t-u)+\sigma_{w_{1}}(t-s) \sigma_{w_{1}+w_{2}}(s-u)=0,
$$


see for example, [FRV].

Fix discriminantal convenient complex numbers $\boldsymbol{w}=\left(w_{1}, \ldots, w_{k}\right)$ and distinct complex numbers $\boldsymbol{z}=\left(z_{1}, \ldots, z_{n}\right)$. For $i=1, \ldots, k$, we say that $t_{i}$ has weight $w_{i}$.

Let $T$ be an admissible forest and $v$ a vertex of $T$. The connected component of $T$, containing $v$, has exactly one vertex, say $z_{a}$, from the set $\left\{z_{1}, \ldots, z_{n}\right\}$. We define the branch $B(v)$ of $v$ to be the collection of those vertices $u$ for which the unique path connecting $u$ with $z_{a}$ contains $v$. By definition $v \in B(v)$. The load $L(v)$ of a vertex $v$ in the forest $T$ is defined to be the sum of the weights of the vertices in $B(v)$.

To an admissible forest $T$ with ordered edges $e_{1}, \ldots, e_{k}$, we assign a closed holomorphic differential $k$-form $\phi_{\theta}(T)$ on $M_{z, \Gamma^{k}}$ by the formula

$$
\phi_{\theta}(T)=\wedge_{i=1}^{k} \sigma_{L\left(h\left(e_{i}\right)\right)}\left(h\left(e_{i}\right)-t\left(e_{i}\right), \tau\right) d\left(h\left(e_{i}\right)-t\left(e_{i}\right)\right) .
$$

Notice that if $\boldsymbol{w}$ are discriminantal convenient, then the load of each vertex $h\left(e_{i}\right)$ does not lie in $\Lambda$ and the form is well-defined.

Theorem 3.2 ([FRV]). Assume that $\boldsymbol{w}$ is discriminantal convenient and $z_{1}, \ldots, z_{n}$ project to distinct points of $E$. Then the assignment $T \mapsto \phi_{\theta}(T)$ defines a representation of $\mathcal{A}_{n}^{k}$ on the space of $k$-forms on $M_{\boldsymbol{z}, \Gamma^{k}}$. The representation is an isomorphism onto its image, denoted by $\mathcal{A}_{\boldsymbol{z}, \Gamma^{k}}^{k}$. Each element of $\mathcal{A}_{\boldsymbol{z}, \Gamma^{k}}^{k}$ descends to a closed holomorphic differential form on $\tilde{M}_{\boldsymbol{z}, \tau}$ with values in $\mathcal{L}_{\boldsymbol{w}}$.

The assignment to a form its cohomology class defines a linear map $\mathcal{A}_{n}^{k} \rightarrow H^{k}\left(\tilde{M}_{\boldsymbol{z}, \tau} ; \mathcal{L}_{\boldsymbol{w}}\right)$. In Section 5 the following theorem will be proved.

Theorem 3.3. Assume that the numbers $\boldsymbol{w}$ are discriminantal convenient and $z_{1}, \ldots, z_{n}$ project to distinct points of $E$. Then the map $\mathcal{A}_{n}^{k} \rightarrow H^{k}\left(\tilde{M}_{\boldsymbol{z}, \tau} ; \mathcal{L}_{\boldsymbol{w}}\right)$ is an isomorphism.

Theorems 3.1 and 3.3 imply the second statement of Theorem 2.1.

According to [SV], $\operatorname{dim} \mathcal{A}_{n}^{k}=\sum_{m_{1}+\cdots+m_{n}=k} m_{1} ! \ldots m_{n}$ ! .

\section{TRANSVERSAl ElliptiC HYPERPLANES}

4.1. Elliptic hyperplanes in $E^{k}$. Denote $\mathcal{E}=E^{k}$. Any $k \times k$-matrix $C \in G L(k, \mathbb{Z})$ defines an isomorphism $\mathcal{E} \rightarrow \mathcal{E},\left(\tilde{t}_{1}, \ldots, \tilde{t}_{k}\right) \mapsto\left(\sum_{j} \tilde{t}_{j} c_{j 1}, \ldots, \sum_{j} \tilde{t}_{j} c_{j k}\right)$. The collection $\sum_{j} \tilde{t}_{j} c_{j 1}, \ldots, \sum_{j} \tilde{t}_{j} c_{j k}$ will be called coordinates on $\mathcal{E}$.

Let $\tilde{t}_{1}^{\prime}, \ldots, \tilde{t}_{k}^{\prime}$ be coordinates on $\mathcal{E}$. Fibers of the projection $\mathcal{E} \rightarrow E^{\ell}$ along the last $k-\ell$ coordinates will be called elliptic $k-\ell$-planes in $\mathcal{E}$, in particular, elliptic $k-1$-planes are elliptic hyperplanes. Fibers of the same projection will be called parallel $k-\ell$-planes.

An elliptic $k-\ell$-plane is defined by equations $\tilde{t}_{i}^{\prime}=\tilde{z}_{i}, i=1, \ldots, \ell$, for suitable $\tilde{z}_{i} \in E$. Each elliptic $k-\ell$-plane is isomorphic to $E^{k-\ell}$ as an algebraic variety.

Lemma 4.1. The normal bundle of an elliptic $k-\ell$-plane in $\mathcal{E}$ is trivial.

4.2. Intersection of $\ell \leqslant k$ transversal elliptic hyperplanes. We say that $\ell \leqslant k$ elliptic hyperplanes $\tilde{H}_{1}, \ldots, \tilde{H}_{\ell}$ intersect transversally, if they are defined by equations

$$
\tilde{t}_{1} a_{1 j}+\cdots+\tilde{t}_{k} a_{k j}-\tilde{z}_{j}=0, \quad j=1, \ldots, \ell,
$$

and the rank of the $\ell \times k$-matrix $a=\left(a_{i j}\right)$ equals $\ell$. 
By standard theorems, see for example [Vi], there are coordinates $\tilde{t}_{1}^{\prime}, \ldots, \tilde{t}_{k}^{\prime}$ on $\mathcal{E}$ such that system (4.1) is equivalent to a system

$$
d_{j} \tilde{t}_{j}^{\prime}-\tilde{z}_{j}^{\prime}=0, \quad j=1, \ldots, \ell,
$$

where $\tilde{z}_{j}^{\prime} \in E, d_{j} \in \mathbb{Z}_{>0}$ and $d_{j} \mid d_{j+1}$ for $j=1, \ldots, \ell-1$. Therefore, the intersection $X$ of $\ell$ transversal hyperplanes in $\mathcal{E}$ consists of $\left(d_{1} \ldots d_{\ell}\right)^{2}$ parallel elliptic $k-\ell$-planes.

Let $\boldsymbol{w}=\left(w_{1}, \ldots, w_{k}\right)$ be complex numbers and $\mathcal{L}_{\boldsymbol{w}}$ the line bundle over $\mathcal{E}$ with a flat connection defined in Section 2. We say that $\boldsymbol{w}$ are convenient for $\mathcal{E}$, if there are no nonzero $\mathcal{L}_{\boldsymbol{w}}$-valued holomorphic differential $k$-forms on $\mathcal{E}$.

Lemma 4.2. Complex numbers $\boldsymbol{w}$ are convenient for $\mathcal{E}$ if and only if $\boldsymbol{w} \notin \Lambda^{k}$.

Proof. It is enough to prove the lemma for $k=1$. If $k=1$ and $\omega$ is an $\mathcal{L}_{\boldsymbol{w}}$-valued holomorphic 1-form, then it is 1-periodic and has the Fourier series expansion. The expansion easily implies the required statement.

Similarly, we say that $\boldsymbol{w}$ are convenient for the transversal intersection $X$ with $\operatorname{dim} X=$ $k-\ell>0$, if there are no nonzero $\mathcal{L}_{\boldsymbol{w}}$-valued holomorphic differential $k-\ell$-forms on any of the parallel $k-\ell$-planes composing $X$.

Lemma 4.3. Complex numbers $\boldsymbol{w}$ are convenient for the transversal intersection $X, \operatorname{dim} X>$ 0 , if and only if there exist integers $l_{1}, \ldots, l_{k}$ such that $\sum_{i=1} l_{i} a_{i j}=0$ for $j=1, \ldots, \ell$, and $l_{1} w_{1}+\cdots+l_{k} w_{k} \notin \Lambda$.

Proof. The proof of Lemma 4.3 is the same as the proof of Lemma 4.2.

Lemma 4.4. If the numbers $\boldsymbol{w}$ are convenient for $\mathcal{E}$, then $H^{*}\left(\mathcal{E}, \mathcal{L}_{\boldsymbol{w}}\right)=0$. If $\boldsymbol{w}$ are convenient for the transversal intersection $X, \operatorname{dim} X>0$, then $H^{*}\left(X,\left.\mathcal{L}_{\boldsymbol{w}}\right|_{X}\right)=0$.

Proof. The lemma follows from the Kunneth formula and the fact that the cohomology of a circle with coefficients in a nontrivial local system is zero.

4.3. Differential forms of $k$ transversal hyperplanes in $\mathcal{E}$. This section contains the main construction of the paper.

Let $k$ transversal elliptic hyperplanes $\tilde{H}_{1}, \ldots, \tilde{H}_{k}$ in $\mathcal{E}$ be given by equations

$$
\sum_{i=1}^{k} \tilde{t}_{i} a_{i j}=\tilde{z}_{j} \quad j=1, \ldots, k,
$$

where $\tilde{t}_{1}, \ldots, \tilde{t}_{k}$ are coordinates on $\mathcal{E}, a=\left(a_{i j}\right)$ is an integer matrix (with nonzero determinant) and $\tilde{z}_{1}, \ldots, \tilde{z}_{k}$ are some points of $E$.

For a complex number $c$, we denote by $\tilde{c}$ its projection to $E$. In particular, $\tilde{0} \in E$ is the projection of 0 . For given complex numbers $\boldsymbol{w}=\left(w_{1}, \ldots, w_{k}\right)$, we consider the system of equations

$$
\sum_{j=1}^{k} a_{i j} \tilde{v}_{j}=\tilde{w}_{i} \quad i=1, \ldots, k,
$$

with respect to the unknown $\tilde{v}_{1}, \ldots, \tilde{v}_{k} \in E$. We say that $\boldsymbol{w}$ is admissible for $\tilde{H}_{1}, \ldots, \tilde{H}_{k}$ if any coordinate $\tilde{v}_{j}$ of any solution of (4.4) is not equal to $\tilde{0}$. 
Lemma 4.5. Assume that the numbers $\boldsymbol{w}$ are convenient for each of the transversal intersections $X_{j}, j=1, \ldots, k$, where $X_{j}$ is the intersection of the elliptic hyperplanes $\tilde{H}_{1}, \ldots, \tilde{H}_{j-1}$, $\tilde{H}_{j+1}, \ldots, \tilde{H}_{k}$, then $\boldsymbol{w}$ are admissible for $\tilde{H}_{1}, \ldots, \tilde{H}_{k}$.

Proof. Let $\tilde{v}_{1}, \ldots, \tilde{v}_{k}$ be any solution of system (4.4). Let $l_{1}, \ldots, l_{k}$ be integers such that $\sum_{i=1} l_{i} a_{i j}=0$ for $j=2, \ldots, k$, and $l_{1} w_{1}+\cdots+l_{k} w_{k} \notin \Lambda$. Then $\tilde{0} \neq \sum_{i} l_{i} \tilde{w}_{i}=\sum_{i j} l_{i} a_{i j} \tilde{v}_{j}=$ $\sum_{i} l_{i} a_{i 1} \tilde{v}_{1}$. Hence $\tilde{v}_{1} \neq \tilde{0}$. Similarly we prove that $\tilde{v}_{2}, \ldots, \tilde{v}_{k}$ are not equal to $\tilde{0}$.

Let $\boldsymbol{w}$ be admissible for $\tilde{H}_{1}, \ldots, \tilde{H}_{k}$. Fix complex numbers $z_{1}, \ldots, z_{k}$ whose projections to $E$ are $\tilde{z}_{1}, \ldots, \tilde{z}_{k}$. For any integers $A_{i}, B_{i}, C_{i}, D_{i}$ with $i=1, \ldots, k$, we consider two systems of equations:

$$
\sum_{i=1}^{k} u_{i} a_{i j}=A_{j} \tau+B_{j}+z_{j}, \quad j=1, \ldots, k
$$

and

$$
\sum_{j=1}^{k} a_{i j} v_{j}=C_{i} \tau+D_{i}+w_{i}, \quad i=1, \ldots, k .
$$

The first system is with respect to complex numbers $\boldsymbol{u}=\left(u_{1}, \ldots, u_{k}\right)$ and the second system is with respect to complex numbers $\boldsymbol{v}=\left(v_{1}, \ldots, v_{k}\right)$.

To the solution $\boldsymbol{v}=\left(v_{1}, \ldots, v_{k}\right)$ of (4.6), we assign the meromorphic $k$-form on $\mathbb{C}^{k}$,

$$
\begin{aligned}
\omega_{\boldsymbol{v}}(\boldsymbol{t}, \tau) & =\omega_{\boldsymbol{v}}\left(t_{1}, \ldots, t_{k}, \tau\right)= \\
& =\operatorname{det} a e^{-2 \pi \sqrt{-1} \sum_{i=1}^{k} C_{i} t_{i}} \prod_{j=1}^{k} \sigma_{v_{j}}\left(\sum_{i=1}^{k} t_{i} a_{i j}-z_{j}, \tau\right) d t_{1} \wedge \cdots \wedge d t_{k} .
\end{aligned}
$$

The form is well-defined since the numbers $\boldsymbol{w}$ are admissible for $\tilde{H}_{1}, \ldots, \tilde{H}_{k}$.

Lemma 4.6. The form $\omega_{\boldsymbol{v}}(\boldsymbol{t}, \tau)$ descends to an $\mathcal{L}_{\boldsymbol{w}}$-valued meromorphic form on $\mathcal{E}$, i.e. $\omega_{\boldsymbol{v}}(\boldsymbol{t}+\gamma, \tau)=\rho_{\boldsymbol{w}}(\gamma) \omega_{\boldsymbol{v}}(\boldsymbol{t}, \tau)$ for $\gamma \in \Gamma^{k}$.

Lemma 4.7. The form $\omega_{\boldsymbol{v}}(\boldsymbol{t}, \tau)$ does not change if $\boldsymbol{v}$ is changed by an element of $\Lambda^{k}$.

Lemma 4.8. Let $\boldsymbol{u}=\left(u_{1}, \ldots, u_{k}\right)$ be the solution of system (4.5). Then

$$
\begin{aligned}
& \omega_{\boldsymbol{v}}\left(t_{1}+u_{1}, \ldots, t_{k}+u_{k}, \tau\right)= \\
& \quad=M(\boldsymbol{u}, \boldsymbol{v}) \operatorname{det} a e^{-2 \pi \sqrt{-1} \sum_{i=1}^{k} C_{i} t_{i}} \prod_{j=1}^{k} \sigma_{v_{j}}\left(\sum_{i=1}^{k} t_{i} a_{i j}, \tau\right) d t_{1} \wedge \cdots \wedge d t_{k},
\end{aligned}
$$

where

$$
M(\boldsymbol{u}, \boldsymbol{v})=e^{2 \pi \sqrt{-1} \sum_{i=1}^{k}\left(A_{i} v_{i}-C_{i} u_{i}\right)}
$$

For a complex number $c$, we shall write $c=c_{\mathbb{R}}+\tau c_{\tau}$ with $c_{\mathbb{R}}, c_{\tau} \in \mathbb{R}$. 
Lemma 4.9. We have

$$
\sum_{i=1}^{k}\left(A_{i} v_{i}-C_{i} u_{i}\right)=\sum_{i=1}^{k}\left(A_{i} v_{i, \mathbb{R}}-B_{i} v_{i, \tau}\right)+\sum_{i=1}^{k}\left(u_{i} w_{i, \tau}-z_{i} v_{i, \tau}\right) .
$$

Proof. $\sum_{i} A_{i} v_{i}=\sum_{i}\left(A_{i} v_{i, \mathbb{R}}+\tau A_{i} v_{i, \tau}\right)$ and $\sum_{i} C_{i} u_{i}=\sum_{i}\left(\sum_{j} a_{i j} v_{j, \tau}-w_{i, \tau}\right) u_{i}=$ $\sum_{i j} u_{i, \mathbb{R}} a_{i j} v_{j, \tau}+\tau \sum_{i j} u_{i, \tau} a_{i j} v_{j, \tau}-\sum_{i} w_{i, \tau} u_{i}=\sum_{j}\left(B_{j}+z_{j, \mathbb{R}}\right) v_{j, \tau}+\tau \sum_{j}\left(A_{j}+z_{j, \tau}\right) v_{j, \tau}-$ $\sum_{i} w_{i, \tau} u_{i}$. These equalities give the lemma.

If $\boldsymbol{u}$ is a solution of (4.5), then $p(\boldsymbol{u})$ is a solution (4.3). All solutions of (4.3) have this form. Similar relations hold for systems (4.6) and (4.4).

Each of the systems (4.3) and (4.4) has ( $\operatorname{det} a)^{2}$ solutions. For each solution $\tilde{\boldsymbol{u}}$ of (4.3) we fix a solution $\boldsymbol{u}$ of (4.5) such that $p(\boldsymbol{u})=\tilde{\boldsymbol{u}}$. We denote by $\mathcal{U}$ the constructed set of $(\operatorname{det} a)^{2}$ points $\boldsymbol{u} \in \mathbb{C}^{k}$. For each solution $\tilde{\boldsymbol{v}}$ of (4.4) we fix a solution $\boldsymbol{v}$ of (4.6) such that $p(\boldsymbol{v})=\tilde{\boldsymbol{v}}$. We denote by $\mathcal{V}$ the constructed set of $(\operatorname{det} a)^{2}$ points $\boldsymbol{v} \in \mathbb{C}^{k}$.

Theorem 4.10. The matrix $M=(M(\boldsymbol{u}, \boldsymbol{v}))_{\boldsymbol{u} \in \mathcal{U}, \boldsymbol{v} \in \mathcal{V}}$ is nondegenerate.

Proof. Let $M_{1}(\boldsymbol{u}, \boldsymbol{v})=e^{2 \pi \sqrt{-1} \sum_{i=1}^{k}\left(A_{i} v_{i, \mathbb{R}}-B_{i} v_{i, \tau}\right)}$. The matrix $M_{1}=\left(M_{1}(\boldsymbol{u}, \boldsymbol{v})\right)_{\boldsymbol{u} \in \mathcal{U}, \boldsymbol{v} \in \mathcal{V}}$ is obtained from $M$ by multiplication by nondegenerate diagonal matrices. Thus, it is enough to prove that $M_{1}$ is nondegenerate.

The nondegeneracy of $M_{1}$ follows from the nondegeneracy of $M_{1}$ for $\boldsymbol{w}=0$ and $\boldsymbol{z}=0$, since the matrix $M_{1}$ for $\boldsymbol{w}, \boldsymbol{z}$ not necessarily equal to zero is obtained from the matrix $M_{1}$ with $\boldsymbol{w}=0, \boldsymbol{z}=0$ by multiplication by nondegenerate diagonal matrices.

By elementary row and column transformations, the pair of systems (4.5) and (4.6) can be reduced to the case of a diagonal matrix $a$. For a diagonal $a$ and $\boldsymbol{w}=0, \boldsymbol{z}=0$ the nondegeneracy of $M_{1}$ is obvious.

Theorem 4.11. Let $\boldsymbol{w}$ be admissible for $\tilde{H}_{1}, \ldots, \tilde{H}_{k}$. Let $\mathcal{U}$ be a set as above. Then there exist the unique differential $k$-forms $\omega_{\boldsymbol{u}, \tilde{H}_{1}, \ldots, \tilde{H}_{k}}(\boldsymbol{t}, \tau), \boldsymbol{u} \in \mathcal{U}$, such that each $\omega_{\boldsymbol{u}, \tilde{H}_{1}, \ldots, \tilde{H}_{k}}(\boldsymbol{t}, \tau)$ is a $\mathbb{C}$-linear combination of forms $\omega_{\boldsymbol{v}}(\boldsymbol{t}, \tau), \boldsymbol{v} \in \mathcal{V}$, and for any $\boldsymbol{u}, \boldsymbol{u}^{\prime} \in \mathcal{U}$ we have the followings expansion,

$$
\omega_{\boldsymbol{u}, \tilde{H}_{1}, \ldots, \tilde{H}_{k}}\left(\boldsymbol{t}+\boldsymbol{u}^{\prime}, \tau\right)=\left(\delta_{\boldsymbol{u}, \boldsymbol{u}^{\prime}}+\mathcal{O}(\boldsymbol{t})\right) d \log \left(\sum_{i=1}^{k} t_{i} a_{i 1}\right) \wedge \cdots \wedge d \log \left(\sum_{i=1}^{k} t_{i} a_{i k}\right),
$$

where $\mathcal{O}(\boldsymbol{t})$ is a function holomorphic at $\boldsymbol{t}=0$ and $\mathcal{O}(\mathbf{0})=0$.

Proof. The theorem is a direct corollary of Theorem 4.10 .

Given transversal $\tilde{H}_{1}, \ldots, \tilde{H}_{k}$, the set $\mathcal{U}$ is not unique, each point $\boldsymbol{u}^{\prime} \in \mathcal{U}$ can be shifted by any element $\gamma=\left(l_{1} \tau+m_{1}, \ldots, l_{k} \tau+m_{k}\right)$ of $\Lambda^{k}$.

Lemma 4.12. Assume that exactly one point $\boldsymbol{u}^{\prime}$ of the set $\mathcal{U}$ is replaced with a point $\boldsymbol{u}^{\prime \prime}=$ $\boldsymbol{u}^{\prime}+\gamma$. Consider the set of differential forms assigned to the new set $\mathcal{U}$ by Theorem 4.11. Then $\omega_{\boldsymbol{u}^{\prime \prime}, \tilde{H}_{1}, \ldots, \tilde{H}_{k}}(\boldsymbol{t}, \tau)=e^{2 \pi \sqrt{-1}\left(w_{1} l_{1}+\cdots+w_{k} l_{k}\right)} \omega_{\boldsymbol{u}^{\prime}, \tilde{H}_{1}, \ldots, \tilde{H}_{k}}(\boldsymbol{t}, \tau)$ and all other differential forms $\omega_{\boldsymbol{u}, \tilde{H}_{1}, \ldots, \tilde{H}_{k}}(\boldsymbol{t}, \tau), \boldsymbol{u} \in \mathcal{U}$, remain unchanged. 
4.3.1. The residue of $\omega_{\boldsymbol{u}, \tilde{H}_{1}, \ldots, \tilde{H}_{k}}$. Let $H$ be a hyperplane in $\mathbb{C}^{k}$ defined by an equation $t_{1} a_{1 j}+\cdots+t_{k} a_{k j}=A_{j} \tau+B_{j}+z_{j}$, where $j \in\{1, \ldots, k\}$ and $A_{j}, B_{j}$ are some integers, c.f. (4.5) and (4.7). We have $p(H)=\tilde{H}_{j}$. Let $\boldsymbol{u}$ be a point of $\mathcal{U}$ and $\omega_{\boldsymbol{u}, \tilde{H}_{1}, \ldots, \tilde{H}_{k}}$ the corresponding differential form. We denote by $\eta_{\boldsymbol{u}}$ the residue of $\omega_{\boldsymbol{u}, \tilde{H}_{1}, \ldots, \tilde{H}_{k}}$ at $H$.

Lemma 4.13. Assume that a vector $\gamma=\left(l_{1} \tau+m_{1}, \ldots, l_{k} \tau+m_{k}\right) \in \Lambda^{k}$ is tangent to $H$, i.e. $\sum_{i}\left(l_{i} \tau+m_{i}\right) a_{i j}=0$. Then for all $\boldsymbol{t} \in H$, we have $\eta_{\boldsymbol{u}}(\boldsymbol{t}+\gamma)=e^{2 \pi \sqrt{-1}\left(w_{1} l_{1}+\cdots+w_{k} l_{k}\right)} \eta_{\boldsymbol{u}}(\boldsymbol{t})$. That is, the form $\eta_{\boldsymbol{u}}$ defines an $\mathcal{L}_{\boldsymbol{w}}$-valued differential form over the elliptic hyperplane $p(H)=\tilde{H}_{j} \subset \mathcal{E}$.

Now we choose $H$ in Lemma 4.13 so that $\boldsymbol{u} \in H$.

For $i \neq j$, the intersection $\tilde{H}_{i} \cap \tilde{H}_{j}$ is a collection of parallel elliptic $k-2$-planes. We denote by $\tilde{H}_{i}^{(j)}$ that elliptic $k-2$-plane which contains $\tilde{\boldsymbol{u}}=p(\boldsymbol{u})$. Then $\tilde{H}_{1}^{(j)}, \ldots, \tilde{H}_{j-1}^{(j)}, \tilde{H}_{j+1}^{(j)}, \ldots, \tilde{H}_{k}^{(j)}$ are transversal elliptic hyperplanes in $\tilde{H}_{j}$.

Theorem 4.14. We have $\eta_{\boldsymbol{u}}(\boldsymbol{t})=(-1)^{j-1} \omega_{\boldsymbol{u}, \tilde{H}_{1}^{(j)}, \ldots, \tilde{H}_{j-1}^{(j)}, \tilde{H}_{j+1}^{(j)}, \ldots, \tilde{H}_{k}^{(j)}}$.

Proof. The difference of the right hand side and the left hand side defines an $\mathcal{L}_{\boldsymbol{w}}$-valued form on $\tilde{H}_{j}$ with logarithmic singularities along $\tilde{H}_{1} \cap \tilde{H}_{j}, \ldots, \tilde{H}_{j-1} \cap \tilde{H}_{j}, \tilde{H}_{j+1} \cap \tilde{H}_{j}, \ldots, \tilde{H}_{k} \cap \tilde{H}_{j}$. The difference has zero $k$-1-iterated residues at all points. Therefore, the difference vanishes due to the following lemma.

Lemma 4.15. Assume that for every $i=1, \ldots, k$, we have a finite set of parallel hyperplanes $\left\{\tilde{H}_{i}^{l_{i}} \mid l_{i} \in L_{i}\right\}$ in $\mathcal{E}$. Assume that the hyperplanes $\tilde{H}_{1}^{l_{1}}, \ldots, \tilde{H}_{k}^{l_{k}}$ intersect transversally. Assume that numbers $\boldsymbol{w}$ are convenient for the transversal intersection of $\tilde{H}_{1}^{l_{1}}, \ldots, \tilde{H}_{k}^{l_{k}}$.

Let $\Omega$ be an $\mathcal{L}_{\boldsymbol{w}}$-valued meromorphic differential $k$-form on $\mathcal{E}$ with logarithmic singularities at the union of all hyperplanes $\left\{\tilde{H}_{i}^{l_{i}} \mid i=1, \ldots, k, l_{i} \in L_{i}\right\}$. Assume that $\Omega$ has zero $k$-iterated residues at all points of $\mathcal{E}$. Then $\Omega$ is the zero form.

Proof. The proof is by induction on $k$. If $k=1$, then $\Omega$ is regular on $E$. Since $\boldsymbol{w}$ are convenient, $\Omega$ vanishes.

Step of the induction. The residue of $\Omega$ at any hyperplane $\tilde{H}_{j}^{l_{j}}$ has the same properties as $\Omega$ : the residue has logarithmic singularities at the union of all intersections $\tilde{H}_{i}^{l_{i}} \cap \tilde{H}_{j}^{l_{j}}$, the residue has zero $k-2$-iterated residue at any point. By the induction assumption, the residue of $\Omega$ at $\tilde{H}_{j}^{l_{j}}$ vanishes, hence, $\Omega$ is regular on $\mathcal{E}$ and $\Omega$ is the zero form due to the convenience of $\boldsymbol{w}$.

4.3.2. Example. Here is an example illustrating Theorem 4.11 for $k=1$. Consider an analog of the pair of systems (4.3) and (4.4): $2 \tilde{t}=0$ and $2 \tilde{v}=\tilde{w}$, where $w \notin \Lambda$. We can choose $\mathcal{U}=\{0,1 / 2, \tau / 2,1 / 2+\tau / 2\}$ and $\mathcal{V}=\{w / 2, w / 2+1 / 2, w / 2+\tau / 2, w / 2+1 / 2+\tau / 2\}$. The differential forms $\omega_{\boldsymbol{v}}, \boldsymbol{v} \in \mathcal{V}$, given by formula (4.7), are

$$
\begin{array}{rlrl}
\omega_{1} & =2 \sigma_{w / 2}(2 t, \tau) d t, & \omega_{2} & =2 \sigma_{w / 2+1 / 2}(2 t, \tau) d t, \\
\omega_{3}=2 e^{-2 \pi \sqrt{-1} t} \sigma_{w / 2+\tau / 2}(2 t, \tau) d t, & \omega_{4}=2 e^{-2 \pi \sqrt{-1} t} \sigma_{w / 2+1 / 2+\tau / 2}(2 t, \tau) d t .
\end{array}
$$

Denote $\gamma=e^{-\pi \sqrt{-1} w}$. Then the differential forms $\omega_{\boldsymbol{u}}, \boldsymbol{u} \in \mathcal{U}$, given by Theorem 4.11, are

$$
\tilde{\omega}_{1}=\frac{1}{4}\left(\omega_{1}+\omega_{2}+\gamma \omega_{3}+\gamma \omega_{4}\right), \quad \tilde{\omega}_{2}=\frac{1}{4}\left(\omega_{1}+\omega_{2}-\gamma \omega_{3}-\gamma \omega_{4}\right),
$$




$$
\tilde{\omega}_{3}=\frac{1}{4}\left(\omega_{1}-\omega_{2}+\gamma \omega_{3}-\gamma \omega_{4}\right), \quad \tilde{\omega}_{4}=\frac{1}{4}\left(\omega_{1}-\omega_{2}-\gamma \omega_{3}+\gamma \omega_{4}\right) .
$$

The forms $\tilde{\omega}_{i}, i=1, \ldots, 4$, define meromorphic $\mathcal{L}_{\boldsymbol{w}^{-}}$valued differential forms on $E$. The form $\tilde{\omega}_{1}$ is regular on $\mathbb{C}-\Lambda$, has simple poles at $\Lambda$, has residue 1 at $t=0$. The forms $\tilde{\omega}_{2}, \tilde{\omega}_{3}, \tilde{\omega}_{4}$ have similar properties with respect to the sets $\mathbb{C}-(1 / 2+\Lambda), \mathbb{C}-(\tau / 2+\Lambda), \mathbb{C}-(\tau / 2+1 / 2+\Lambda)$ and points $1 / 2, \tau / 2, \tau / 2+1 / 2$, respectively. These properties imply that

$$
\begin{aligned}
\tilde{\omega}_{1}=\sigma_{w}(t, \tau) d t, & \tilde{\omega}_{2}=\sigma_{w}(t-1 / 2, \tau) d t, \\
\tilde{\omega}_{3}=\sigma_{w}(t-\tau / 2, \tau) d t, & \tilde{\omega}_{4}=\sigma_{w}(t-\tau / 2-1 / 2, \tau) d t .
\end{aligned}
$$

\section{ARBitrary ELLIPTIC ARRANGEMENT}

5.1. An elliptic arrangement. An elliptic arrangement in $\mathcal{E}=E^{k}$ is a finite collection $\mathcal{C}=\left\{\tilde{H}_{j}\right\}_{j \in J}$ of elliptic hyperplanes. We fix coordinates $\tilde{t}_{1}, \ldots, \tilde{t}_{k}$ on $\mathcal{E}$ and for every $j \in J$ we fix an equation $\tilde{t}_{1} a_{1 j}+\cdots+\tilde{t}_{k} a_{k j}-\tilde{z}_{j}=0$ defining the hyperplane $\tilde{H}_{j}$.

We denote by

$$
\tilde{M}_{\mathcal{C}}=\mathcal{E}-\cup_{j \in J} \tilde{H}_{j}
$$

the complement of the arrangement.

Consider the intersection of any $\ell \leqslant k$ transversal hyperplanes of $\mathcal{C}$. The intersection consists of a finite set of parallel elliptic $k-\ell$ planes. Each of these $k-\ell$-planes will be called an edge of $\mathcal{E}$. In particular, if $\ell=k$, then the 0 -planes will be called vertices of $\mathcal{E}$.

For an edge $X$ we denote $J_{X}=\left\{j \in \subset J \mid X \subset \tilde{H}_{j}\right\}$.

We denote by $\tilde{\mathcal{U}}$ the set of all vertices of $\mathcal{C}$. For every vertex $\tilde{\boldsymbol{u}} \in \tilde{\mathcal{U}}$ we choose a point $\boldsymbol{u} \in \mathbb{C}^{k}$ such that $p(\boldsymbol{u})=\tilde{\boldsymbol{u}}$. The set of all chosen points in $\mathbb{C}^{k}$ is denoted by $\mathcal{U}$.

We say that complex numbers $\boldsymbol{w}=\left(w_{1}, \ldots, w_{k}\right)$ are convenient for the elliptic arrangement $\mathcal{C}$, if $\boldsymbol{w}$ are convenient for the intersection of every $\ell<k$ transversal hyperplanes $\tilde{H}_{j_{1}}, \ldots, \tilde{H}_{j_{\ell}}$ of $\mathcal{C}$ (in the sense of Section 4.2).

5.2. Differential $k$-forms of an elliptic arrangement. For a vertex $\tilde{\boldsymbol{u}} \in \tilde{\mathcal{U}}$, we denote by $\mathcal{C}_{\tilde{\boldsymbol{u}}}=\left\{\tilde{H}_{j}\right\}_{j \in I_{\tilde{\boldsymbol{u}}}}$ the subarrangement of all hyperplanes of $\mathcal{C}$ containing $\tilde{\boldsymbol{u}}$. In a small neighborhood of $\tilde{\boldsymbol{u}}$ the arrangement $\mathcal{C}_{\tilde{\boldsymbol{u}}}$ is isomorpic to a central arrangement of affine hyperplanes. We denote by $\mathcal{A}_{\tilde{\boldsymbol{u}}}^{k}$ the $k$-th graded component of the Orlik-Solomon algebra of that arrangement. More precisely, let $\mathcal{A}_{\tilde{\boldsymbol{u}}}^{k}$ be the complex vector space generated by symbols $\left(\tilde{H}_{j_{1}}, \ldots, \tilde{H}_{j_{k}}\right)$ with $j_{i} \in J_{\tilde{\boldsymbol{u}}}$, subject to the relations:

(i) $\left(\tilde{H}_{j_{1}}, \ldots, \tilde{H}_{j_{k}}\right)=0$ if $\tilde{H}_{j_{1}}, \ldots, \tilde{H}_{j_{k}}$ are not transversal;

(ii) $\left(\tilde{H}_{j_{\sigma(1)}}, \ldots, \tilde{H}_{j_{\sigma(k)}}\right)=(-1)^{|\sigma|}\left(\tilde{H}_{j_{1}}, \ldots, \tilde{H}_{j_{k}}\right)$ for any $\sigma \in S_{k}$;

(iii) $\sum_{i=1}^{k+1}(-1)^{i}\left(\tilde{H}_{j_{1}}, \ldots, \widehat{\tilde{H}}_{j_{i}}, \ldots, \tilde{H}_{j_{k+1}}\right)=0$ for any $k+1$ elliptic hyperplanes of $\mathcal{C}_{\tilde{\boldsymbol{u}}}$.

We set

$$
\mathcal{A}_{\mathcal{C}}^{k}=\oplus_{\tilde{\boldsymbol{u}} \in \tilde{\mathcal{U}}} \mathcal{A}_{\tilde{\boldsymbol{u}}}^{k}
$$

Let us fix $\boldsymbol{w}=\left(w_{1}, \ldots, w_{k}\right)$ convenient for $\mathcal{E}$. Let $\tilde{\boldsymbol{u}} \in \tilde{\mathcal{U}}$ and $\boldsymbol{u} \in \mathcal{U}$ be such that $p(\boldsymbol{u})=\tilde{\boldsymbol{u}}$. Let $\tilde{H}_{j_{l}}, \ldots, \tilde{H}_{j_{k}}$ be any $k$ transversal hyperplanes in $\mathcal{C}_{\tilde{\boldsymbol{u}}}$. Denote by $\omega_{\boldsymbol{u}, \tilde{H}_{j_{l}}, \ldots, \tilde{H}_{j_{k}}}(\boldsymbol{t}, \tau)$ the differentail meromorphic $k$-form on $\mathbb{C}^{k}$ assigned by Theorem 4.11 to these $k$ transversal hyperplanes and denoted by $\omega_{\boldsymbol{u}, \tilde{H}_{1}, \ldots, \tilde{H}_{k}}(\boldsymbol{t}, \tau)$ in Theorem 4.11. We denote by $A_{\tilde{\boldsymbol{u}}}^{k}$ the complex 
vector space generated by the forms $\omega_{\boldsymbol{u} ; \tilde{H}_{j_{l}}, \ldots, \tilde{H}_{j_{k}}}(\boldsymbol{t}, \tau)$. Notice that by Lemma 4.12, the space $A_{\tilde{\boldsymbol{u}}}^{k}$ does not depend on the choice of $\boldsymbol{u} \operatorname{such} p(\boldsymbol{u})=\tilde{\boldsymbol{u}}$.

We denote by $A_{\mathcal{C}}^{k}$ the sum of vector spaces $A_{\tilde{\boldsymbol{u}}}^{k}, \tilde{\boldsymbol{u}} \in \tilde{\mathcal{U}}$.

\section{Theorem 5.1.}

(i) The map $\mathcal{A}_{\tilde{\boldsymbol{u}}}^{k} \rightarrow A_{\tilde{\boldsymbol{u}}}^{k},\left(\tilde{H}_{j_{l}}, \ldots, \tilde{H}_{j_{k}}\right) \mapsto \omega_{\boldsymbol{u} ; \tilde{H}_{j_{l}}, \ldots, \tilde{H}_{j_{k}}}(\boldsymbol{t}, \tau)$, is an isomorphism of vector spaces.

(ii) We have $A_{\mathcal{C}}^{k}=\oplus_{\tilde{\boldsymbol{u}} \in \tilde{\mathcal{U}}} A_{\tilde{\boldsymbol{u}}}^{k}$.

Proof. It is enough to prove that for any $k+1$ elliptic hyperplanes of $\mathcal{C}_{\tilde{\boldsymbol{u}}}$, we have the elliptic Orlik-Solomon relation

$$
\sum_{i=1}^{k+1}(-1)^{i} \omega_{\boldsymbol{u}, \tilde{H}_{j_{1}}, \ldots, \widehat{\tilde{H}_{i}}, \ldots, \tilde{H}_{j_{k+1}}}=0
$$

The proof is by induction on $k$. If $k=1$, the difference $\omega_{\boldsymbol{u}, \tilde{H}_{1}}-\omega_{\boldsymbol{u}, \tilde{H}_{2}}$ is regular on $E$ and is the zero 1-form due to the convenience of $\boldsymbol{w}$.

Step of the induction. For every $i=1, \ldots, k+1$, the residue at $\tilde{H}_{j_{i}}$ of the left hand side in (5.1) is the left hand side of an elliptic Orlik-Solomon relation for an arrangement in $\tilde{H}_{j_{i}}$, see Theorem 4.14. By the induction assumption, the residue of the left hand side at $\tilde{H}_{j_{i}}$ is the zero $k-1$-form. Hence, the left hand side in (5.1) is regular on $\mathcal{E}$ and vanishes due to the convenience of $\boldsymbol{w}$.

5.3. Cohomology of the complement. Every form $\omega \in A_{\mathcal{C}}^{k}$ induces a holomorphic $\mathcal{L}_{\boldsymbol{w}}$-valued $k$-form $p_{*}(\omega)$ on the complement $\tilde{M}_{\mathcal{C}}$ of the elliptic arrangement $\mathcal{C}$. The image of $A_{\mathcal{C}}^{k}$ will be denoted by $p_{*}\left(A_{\mathcal{C}}^{k}\right)$. The assignment to $p_{*}(\omega)$ its cohomology class $\left[p_{*}(\omega)\right]$ defines a linear map $\iota: A_{\mathcal{C}}^{k} \rightarrow H^{k}\left(\tilde{M}_{\mathcal{C}} ; \mathcal{L}_{w}\right)$. Here $H^{*}\left(\tilde{M}_{\mathcal{C}} ; \mathcal{L}_{\boldsymbol{w}}\right)$ denotes the cohomology of $\tilde{M}_{\mathcal{C}}$ with coefficients in the local system of horizontal sections of $\mathcal{L}_{\boldsymbol{w}}$.

Theorem 5.2. Assume that $\boldsymbol{w}$ are convenient for $\mathcal{C}$. Then $H^{\ell}\left(\tilde{M}_{\mathcal{C}} ; \mathcal{L}_{\boldsymbol{w}}\right)=0$ for $\ell \neq k$ and $\iota_{\mathcal{C}}: A_{\mathcal{C}}^{k} \rightarrow H^{k}\left(\tilde{M}_{\mathcal{C}} ; \mathcal{L}_{\boldsymbol{w}}\right)$ is an isomorphism.

Proof. We need the following lemmas.

Lemma 5.3. The map $\iota_{\mathcal{C}}: A_{\mathcal{C}}^{k} \rightarrow H^{k}\left(\tilde{M}_{\mathcal{C}} ; \mathcal{L}_{\boldsymbol{w}}\right)$ is a monomorphism.

Proof. For a central affine arrangement of hyperplanes in $\mathbb{C}^{k}$, the $k$-th homology group of the complement with trivial coefficients is generated by $k$-dimensional tori located near the vertex of the arrangement and corresponding to the $k$-flags of the arrangement, see Section 4.4 in [SV]. The nondegenerate pairing between the top degree cohomology of the complement with trivial coefficients and the top degree homology is given by the integrals of the Orlik-Solomon differential forms over the tori. The integrals are nothing else but the multiple residues of the differential forms at the flags of the arrangement. Locally at $\tilde{\boldsymbol{u}} \in \tilde{\mathcal{U}}$, the arrangement $\mathcal{C}_{\tilde{\boldsymbol{u}}}$ is isomorphic to a central affine arrangement. The $k$-dimensional tori of that central arrangement, considered as $k$-dimensional tori in a small neighborhood of $\tilde{\boldsymbol{u}}$ in $\tilde{M}$ induces a vector subspace $H_{\tilde{\boldsymbol{u}}, k} \subset H_{k}\left(\tilde{M} ; \mathcal{L}_{\boldsymbol{w}}\right)$. The pairing between $H_{\tilde{\boldsymbol{u}}, k}$ and $A_{\tilde{\boldsymbol{u}}^{\prime}}$ is zero if $\tilde{\boldsymbol{u}} \neq \tilde{\boldsymbol{u}}^{\prime}$ and the pairing is nondegenerate if $\tilde{\boldsymbol{u}}=\tilde{\boldsymbol{u}}^{\prime}$.

Lemma 5.4. Theorem 5.2 is true for $k=1$. 
Proof. The lemma follows from the convenience of $\boldsymbol{w}$ and the exact sequence for the pair $\tilde{M}_{\mathcal{C}} \subset E$.

Let $j_{0}$ be an element of $J$. We consider the following three elliptic arrangements: $\mathcal{C}, \mathcal{C}^{\prime}, \mathcal{C}^{\prime \prime}$, where $\mathcal{C}^{\prime}=\left\{\tilde{H}_{j}\right\}_{j \in J-\left\{j_{0}\right\}}$ and $\mathcal{C}^{\prime \prime}$ is the elliptic arrangement induced by $\mathcal{C}$ on $\tilde{H}_{j_{0}}$.

Lemma 5.5. We have an exact sequence

$$
0 \rightarrow A_{\mathcal{C}^{\prime}}^{k} \rightarrow A_{\mathcal{C}}^{k} \rightarrow A_{\mathcal{C}^{\prime \prime}}^{k-1} \rightarrow 0
$$

where the second map is the residue at $\tilde{H}_{j_{0}}$.

Proof. The lemma follows from the fact that $A_{\mathcal{C}}^{k}, A_{\mathcal{C}^{\prime}}^{k}, A_{\mathcal{C}^{\prime \prime}}^{k-1}$ are isomorphic to the top degree components of the Orlik-Solomon algebras of central arrangements.

\section{Lemma 5.6.}

(i) For $\ell \neq k$ we have $H^{\ell}\left(\tilde{M}_{\mathcal{C}^{\prime}} ; \mathcal{L}_{\boldsymbol{w}}\right)=H^{\ell}\left(\tilde{M}_{\mathcal{C}} ; \mathcal{L}_{\boldsymbol{w}}\right)=H^{\ell-1}\left(\tilde{M}_{\mathcal{C}^{\prime \prime}} ; \mathcal{L}_{\boldsymbol{w}}\right)=0$.

(ii) Consider the following diagram

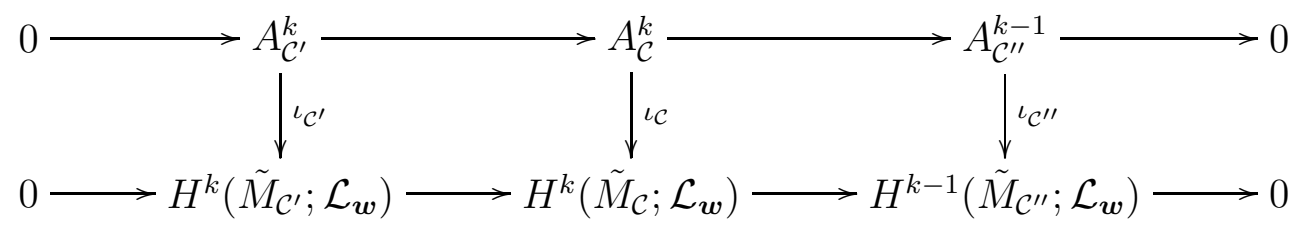

where the top horizontal sequence is the sequence (5.2), the homomorphisms of the bottom horizontal sequence are the homomorphisms of the exact sequence of the pair $\tilde{M}_{\mathcal{C}} \subset \tilde{M}_{\mathcal{C}^{\prime}}$, c.f. Lemma [4.1. Then the diagram is commutative, the horizontal sequence is exact and the vertical homomorphisms are isomorphisms.

Proof. The proof of this lemma is similar to the corresponding proofs in Section 5.4 of [OT]. Namely, using Lemma 4.1, one proves that there is a cohomology long exact sequence

$$
\cdots \rightarrow H^{\ell}\left(\tilde{M}_{\mathcal{C}^{\prime}} ; \mathcal{L}_{\boldsymbol{w}}\right) \rightarrow H^{\ell}\left(\tilde{M}_{\mathcal{C}} ; \mathcal{L}_{\boldsymbol{w}}\right) \rightarrow H^{\ell-1}\left(\tilde{M}_{\mathcal{C}^{\prime \prime}} ; \mathcal{L}_{\boldsymbol{w}}\right) \rightarrow H^{\ell+1}\left(\tilde{M}_{\mathcal{C}^{\prime}} ; \mathcal{L}_{\boldsymbol{w}}\right) \rightarrow \ldots
$$

c.f. Corollary 5.81 in $\left[\mathrm{OT}\right.$. Using the induction on $k$, one proves that $H^{\ell}\left(\tilde{M}_{\mathcal{C}^{\prime}} ; \mathcal{L}_{\boldsymbol{w}}\right) \simeq$ $H^{\ell}\left(\tilde{M}_{\mathcal{C}} ; \mathcal{L}_{\boldsymbol{w}}\right)$ if $\ell \neq k$. Using the induction on the number of hyperplanes in $\mathcal{C}$, one concludes that $H^{\ell}\left(\tilde{M}_{\mathcal{C}} ; \mathcal{L}_{\boldsymbol{w}}\right)=0$ if $\ell \neq k$ and one gets an exact sequence $0 \rightarrow H^{k}\left(\tilde{M}_{\mathcal{C}^{\prime}} ; \mathcal{L}_{\boldsymbol{w}}\right) \rightarrow$ $H^{k}\left(\tilde{M}_{\mathcal{C}} ; \mathcal{L}_{\boldsymbol{w}}\right) \rightarrow H^{k-1}\left(\tilde{M}_{\mathcal{C}^{\prime \prime}} ; \mathcal{L}_{\boldsymbol{w}}\right) \rightarrow 0$. Then using the double induction on $k$ and the number of hyperplanes in $\mathcal{C}$ one gets the second statement of Lemma 5.6.

Lemma 5.6 implies Theorem 5.2 .

Theorem 5.2 implies Theorems 3.3 and 2.1 .

\section{REFERENCES}

[A] V. I. Arnold. The cohomology ring of the pure braid group. Mat. Zametki 5 (1969), 227-231, Math. Notes 5 (1969) 138-140.

[FV1] G. Felder, A. Varchenko. Integral representation of solutions of the elliptic Knizhnik-ZamolodchikovBernard equations. Int. Math. Res. Notices, (N. 5):221-233, 1995.

[FV2] G. Felder, A. Varchenko. Three formulae for eigenfunctions of integrable Schrödinger operators Compositio Math., 107: 143-175, 1997. 
[FRV] G. Felder, R. Rimnyi, A. Varchenko, A. Poincar-Birkhoff-Witt expansions of the canonical elliptic differential form. Quantum groups, 191-208, Contemp. Math., 433, Amer. Math. Soc., Providence, RI, 2007.

[OS] P. Orlik, L. Solomon, Combinatorics and topology of complements of hyperplanes. Invent. Math. 56, 167-189 (1980).

[OT] P. Orlik, H. Terao, Arrangements of Hyperplanes. Springer-Verlag, Berlin-Heidelberg-New York, 1992.

[SV] V.Schechtman, A. Varchenko. Arrangements of hyperplanes and Lie algebra homology. Invent. Math., 106(1):139-194, 1991.

[Vi] E.B. Vinberg A course in algebra. AMS, 2003

[WW] E. T. Whittaker, G. N. Watson. A Course of Modern Analysis. Reprint of the fourth (1927) edition. Cambridge University Press (September 1996). 